\title{
Polyneuropathy due to polyangiitis overlap syndrome
}

\author{
LHTHS KORTBEEK, MWPM VAN BEEK \\ From St Joseph Hospital, Eindhoven, The Netherlands
}

SUMMARY A patient presented with a mild polyneuropathy, developed a large intra-abdominal haemorrhage and died of cardiac arrest within a few days. Microscopic examination revealed a polyangiitis overlap syndrome (Fauci) with lesions in many organs, including the peripheral nerves and the heart. A myocarditis was considered to be the cause of death.

In a case of polyneuropathy many diseases and syndromes are to be considered as possible causes. We describe a type of vasculitis, that has not been mentioned as a cause of polyneuropathy in the literature.

\section{Case report}

A 58-year-old man was admitted with a diagnosis of polyneuropathy. Nine days previously a tingling sensation has started in the right hand and gradually extended to both hands, lower legs and feet. Grip strength in the right hand had diminished. On either side below the mandible some nodes were slightly enlarged with some pain in the neck. There were no other complaints. The patient had been taking $2.5 \mathrm{mg}$ predinose tid for asthmatic bronchitis, and $250 \mathrm{mg}$ methyldopa tid with $50 \mathrm{mg}$ hydrochlorthiazide and $5 \mathrm{mg}$ amiloride hydrochloride per day for three years. Examination revealed a man who walked with difficulty but without cane or other aid, and who did not impress as being very ill. The Romberg test was positive. The patient could not walk a line with the eyes open and could hardly walk on heels and toes. There was no evident loss of strength in the hands. There was no disturbance of sensibility. Tendon reflexes were absent, except for symmetrical weakly positive biceps and triceps. Plantar reflexes were indifferent. There were no trophic disturbances of distal or other muscles, and no other abnormal neurological findings.

The day after admission, while straining for defaecation, violent pain in the abdomen and shock developed with low blood pressure and haemoglobin diminishing from 15.8 to $7.5 \mathrm{~g} / \mathrm{dl}$. An emergency laparotomy was performed and a large, right perirenal haematoma (estimated volume $2 \frac{1}{2}-3$ ls) was evacuated. The cause of the haemorrhage was not found, although the large vessels were dissected free and inspected and peroperative angiography was performed.

Address for reprint requests: Dr LHThS Kortbeek, Elzentlaan 46, $5611 \mathrm{LN}$, Eindhoven, The Netherlands.

Received 5 May 1984 and in revised form 12 July 1984. Accepted 21 July 1984
After blood transfusions the blood pressure returned to normal and the $\mathrm{Hb}$ to $11.3 \mathrm{~g} / \mathrm{dl}$. Three hours later the blood pressure fell to $60 / 40 \mathrm{mmHg}$ and cardiac arrest occurred.

The main feature of the laboratory findings was a marked eosinophia. This had been noted three years before and had persisted in varying degrees. On admission $40 \%$ of the leucocytes in the peripheral blood were eosinophlic granulocytes, ESR $16 \mathrm{~mm} / \mathrm{h}$. Other laboratory findings were normal, including serum IgM, IgG and $\operatorname{IgA}$ values and CSF examination.

The postmortem examination revealed only slight macroscopic changes. A few $\mathrm{ml}$ of liquid blood were found in the peritoneal cavity, with extensive haemorrhagic perfusion of the tissues in the right perirenal field of operation. There was some purulent mucus in the trachea and bronchi, especially on the left. No macroscopic changes of the intra-thoracal and intra-abdominal organs were found. An extensive macroscopic and microscopic neuropathological examination (Prof Dr J Slooff) failed to reveal any lesions in the cerebrum, cerebellum, brain stem or spinal cord.

Microscopic examination revealed a vasculitis, with the following characteristics:

Involvement of many organs, particularly the heart, liver, spleen, periadrenal adipose tissue and probably the lungs, but not the skin and kidneys; localisation in the smaller vessels, especially the capillaries and venules as well as a few small arterial branches, but not the muscular and elastic layers of medium-sized and large arteries; infiltration in all layers of the vascular walls and perivascular regions; in some organs the infiltrate extended to adjacent tissues; in the heart there was myocarditis (fig 1); fibrinoid necrosis at several sites in small arterial branches, with positive Movat and PTAH staining; eosinophilia in the infiltrates in the vessel walls and tissues; developmental stage of the lesions: generally recent lesions, but at one site an older lesion in the form of an organised thrombus.

A more extensive haemorrhage was found around a small arterial branch near the right adrenal gland. The perirenal haemorrhage was probably caused by a vascular rupture. A fragment of a peripheral nerve showed a vasculitis with the same characteristics as those found in the other organs (fig 2). 


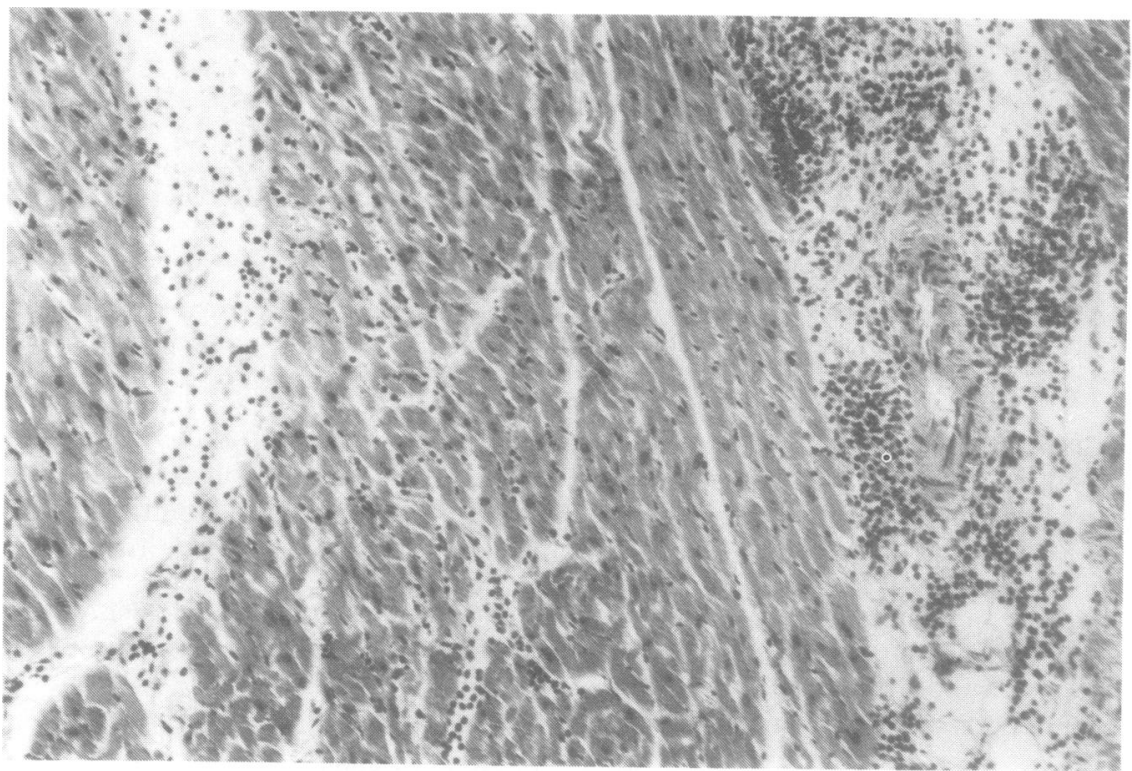

Fig 1 Injiltrate around capillaries, extending into the surrounding myocardium. Movat ( $\times$ 100).

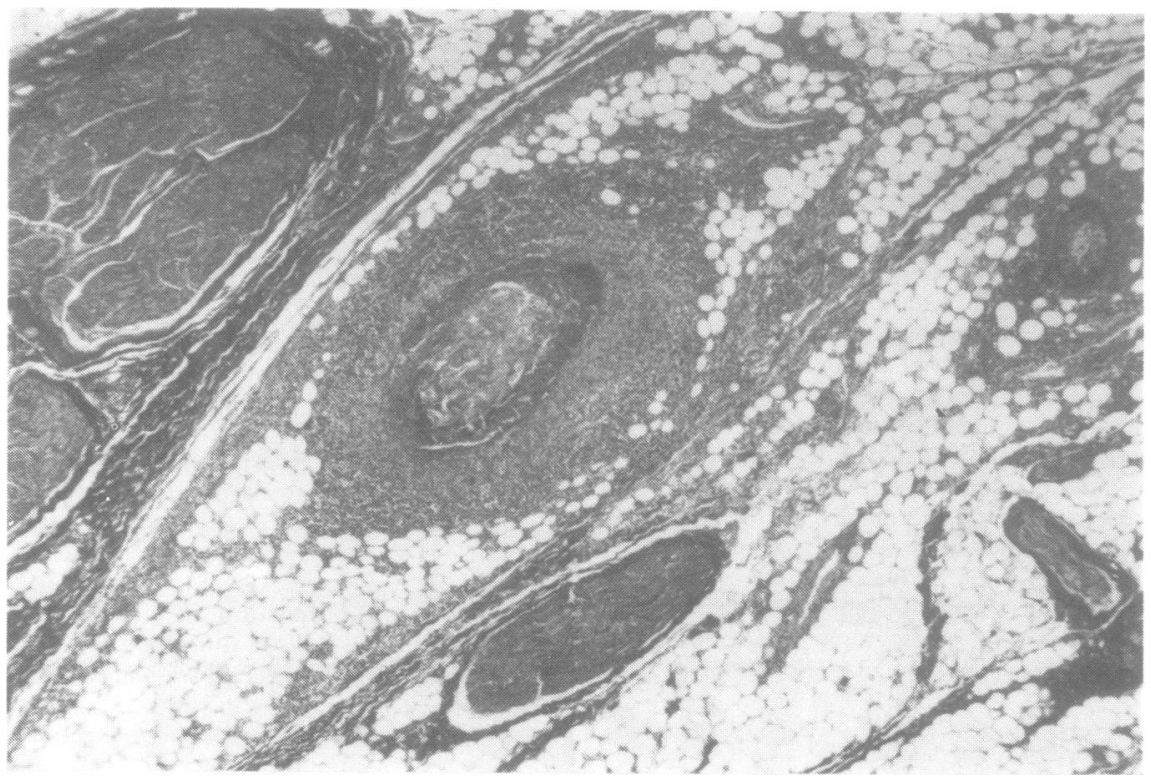

Fig 2 Vasculitis with fibrinoid necrosis of two arterial branches of varying size in a peripheral nerve. PTAH $(\times 40)$. 


\section{Discussion}

The literature comprises some descriptions of vasculitides, especially localised in the small vessels: hypersensitivity vasculitis, ${ }^{2}$ drug related vasculitis ${ }^{3}$ and the polyangiitis overlap syndrome. ${ }^{45}$ In view of the recent developments in the classification of the vasculitides and the limited significance now attached to the term "hypersensitivity vasculitis", the polyangiitis overlap syndrome as defined by Fauci $^{4} 5$ is the most likely choice for the classification of our case. The other forms of vasculitis have other characteristics than those in our case. Polyarteritis nodosa and the Churg-Strauss syndrome (allergic angiitis and granulomatosis) are localised in medium-sized muscular and small arteries, often in circumscribed foci and often with aneurysms. Temporal arteriitis and Wegener's granulomatosis are characterised by giant cells. In our case these features were absent. The clinical features, recently summarised by Berlit et al, ${ }^{6}$ are different too.

Fauci defined the polyangiitis overlap syndrome as a form of necrotising vasculitis involving multiple organ systems, with the prevailing tendency for irreversible organ system dysfunction and with a poor prognosis. The term "overlap" indicates-that this syndrome has some characteristics in common with the polyarteritis nodosa group on the one hand and the hypersensitivity vasculitis (mainly localised in the skin) on the other hand.

Recognition of the true nature of our patient's condition might have had therapeutic consequences. Mullick $e t a^{3}$ studied 30 cases with a clinical hypersensitivity reaction to drugs and a vasculitis. The vasculitis affected only the small vessels and there was marked eosinophilia. Eleven patients had a dermal form with maculo-papular or vesicular eruptions and 19 patients showed a systemic form with involvement of many organs. Fifteen patients died. Among the drugs involved in these cases was chlorothiazide, a component of Moduretic, which was used by our patient. Some drugs of neurological interest are: carbamazepine, diphenylhydantoin, trimethadione. It has not been proven that chlorothiazide did play a role in our case. Nevertheless elimination of possibly causative drugs should have been undertaken if the existence of a vasculitis was suspected. The long-lasting eosinophilia might have been a warning. Faucis has given a detailed account of the therapeutic possibilities in the various forms of vasculitis, including corticosteroids and immunosuppressive drugs.

The neurological literature contains several reports on the relation between drug therapy and affections of the peripheral nervous system and of the musculature..$^{7-11}$ The pathogenesis is seldom specified and the polyangiitis overlap syndrome is not mentioned. The neurologist should be aware of this possibility, particularly if eosinophilia has long existed in a patient on chronic medication.

\section{References}

' Zeek PM. Periarthritis nodosa: a critical review. Am J Clin Pathol 1952;22:777-90.

2 Zeek PM. Periarthritis nodosa and other forms of necrotizing angiitis. New Engl J Med 1953; 18:764-72.

${ }^{3}$ Mullick FG, McAllister HA, Wagnes BM, Fenoglio JJ. Drug related vasculitis; clinicopathologic correlations in 30 patients. Hum Pathol Vol. 10 nr. 3; 1979:31325.

${ }^{4}$ Fauci AS. The spectrum of vasculitis; clinical, pathologic, immunologic and therapeutic considerations. Ann Intern Med 1978;89:660-76.

${ }^{5}$ Fauci AS. Vasculitis. J Allergy Clin Immunol 1983;72:211-23.

- Berlit P, Kessler CH, Storch B, Krause KH. Immunvaskulitis und Nervensystem. Nervenarzt 1983;54:497503.

7 Argov Z, Mastaglia FL. Drug-induced peripheral neuropathies. Br Med J 1979: 1:663-6.

${ }^{8}$ Arnason BGW. Neuropathy of serum sickness. In: Dyck PJ, Thomas PK, Lambert EH, eds. Peripheral Neuropathy, Volume II. London: WB Saunders Company, 1975;55:1104-9.

${ }^{9}$ Conn DL, Dyck PJ. Angiopathic neuropathy in connective tissue diseases. In: Dyck PJ, Thomas PK, Lambert EH, eds. Peripheral Neuropathy, Volume II. London: WB Saunders Company, 1975;57:1149-65.

${ }^{10}$ Lane RJ, Mastaglia FL, Drug-induced myopathies in man. Lancet 1978;ii:562-5.

"Stafford CR, Bogdanoff BM, Green L, Spector HB. Mononeuropathy multiplex as a complication of amphetamine angiitis. Neurology (Minneap) 1975;25:570-2. 